\title{
Um Algoritmo do Tipo Lagrangeano Aumentado para a Correção das Equações do Problema de Fluxo de Potência
}

J.B. FRANCISCO ${ }^{1}$, M.C. ZAMBALDI ${ }^{2}$, Departamento de Matemática, UFSC, 88040-900 Florianópolis, SC, Brasil.

\begin{abstract}
Resumo. O problema de fluxo de potências em engenharia elétrica resulta em um modelo matemático cuja formulação é um sistema de equações não-lineares. Quando ocorre carregamento excessivo nas barras do sistema elétrico, várias equações não apresentam raízes, originando uma formulação de mínimos quadrados nãolineares com uma estrutura bem particular. Neste trabalho abordamos esse modelo por meio de um método do tipo Lagrangeano Aumentado adequado à estrutura particular do problema. Testes numéricos mostram a eficiência da técnica empregada comparada com uma implementação padrão.
\end{abstract}

Palavras-chave. Programação não-linear, Lagrangeano Aumentado, Sistemas de Potências.

\section{Introdução}

Algoritmos robustos e confiáveis para resolver as equações da rede elétrica têm sido frequentemente sugeridos como alternativa ao método de Newton, o qual torna-se inviável em problemas de grande porte [12].

Este trabalho contempla o problema de fluxo de carga com carregamento excessivo. Neste caso, estamos assumindo que o sistema elétrico opera acima de suas limitações. Do ponto de vista matemático isso representa um sistema de equações não-lineares sem solução real.

Baseado em recentes avanços de otimização numérica $[1,5,6,4]$ e tirando proveito da estrutura do problema, este trabalho propõe um método para resolver o problema de fluxo de carga com carregamento excessivo. Vale a pena ressaltar que, em situações onde o sistema opera dentro de suas limitações, o método se resume ao algoritmo de Levenberg-Marquardt para sistemas não-lineares [13].

A abordagem está principalmente baseada no método do Lagrangeano Aumentado e nas idéias de Levenberg-Marquardt, que são empregadas nas iterações internas. Neste caso, sob certas hipóteses, asseguramos convergência a pontos estacionários do problema central.

\footnotetext{
1juliano@mtm.ufsc.br

2 zambaldi@mtm.ufsc.br
} 
O trabalho está dividido como se segue: na Seção 2 apresentamos o problema central, a saber, as equações da rede elétrica. As principais idéias e formulações do problema proposto, bem como as principais teorias envolvidas, são contempladas na Seção 3. Na Seção 4 apresentamos os resultados numéricos em problemas usualmente conhecidos na literatura especializada. Para finalizar, a Seção 4 expõe as principais conclusões deste trabalho.

Notação: $\mathbb{R}^{++}=\{x \in \mathbb{R} \mid x>0\} . \mathbb{N}=\{0,1,2,3, \ldots\}$.

\section{O Problema das Equações do Fluxo de Potência}

Considere um sistema elétrico com $n b$ barras e matriz de admitância dada por $Y=$ $G+j B$, em que $j=\sqrt{-1}$. Os elementos desta matriz são constantes matemáticas e apresentam sua própria representatividade intrínseca no sistema elétrico. Vamos denotar as potências ativa e reativa de demanda, respectivamente, por $P_{i}^{d}$ e $Q_{i}^{d}$, as quais são fornecidas pelo problema. Portanto, definindo $c_{i k}=\cos \left(\theta_{i}-\theta_{k}\right) \mathrm{e}$ $s_{i k}=\operatorname{sen}\left(\theta_{i}-\theta_{k}\right)$, as equações do problema de fluxo de carga em coordenadas polares podem ser representadas pelo seguinte conjunto de equações não-lineares:

$$
\begin{aligned}
P_{i}^{c a l}(V, \theta) & =\left[G_{i i} V_{i}^{2}+V_{i} \sum_{k \in \Omega_{i}} V_{k}\left(G_{i k} c_{i k}+B_{i k} s_{i k}\right)\right]-P_{i}^{d} \\
Q_{i}^{c a l}(V, \theta) & =\left[-B_{i i} V_{i}^{2}+V_{i} \sum_{k \in \Omega_{i}} V_{k}\left(G_{i k} s_{i k}+B_{i k} c_{i k}\right)\right]-Q_{i}^{d}
\end{aligned}
$$

em que $i=1, \ldots, n b$. As variáveis do problema são $V \in \mathbb{R}^{n b}$ e $\theta \in \mathbb{R}^{n b}$, que representam, respectivamente, a magnitude de tensão e o ângulo de tensão nodal. $\mathrm{O}$ conjunto de índices $\Omega_{i}$ define a topologia do sistema, isto é, a conexão da barra $i$ com as demais, que por sua vez influencia diretamente a estrutura esparsa das matrizes associadas ao problema. Maiores detalhes desta formulação podem ser obtidos em [12].

Em sistemas elétricos com carregamento excessivo várias equações do sistema acima não se realizam, dando origem a um problema de mínimos quadrados nãolinear. Em virtude de solicitações do sistema elétrico, algumas equações devem efetivamente se realizarem, sendo, portanto, incorporadas como restrições de igualdade no problema. Nesta formulação, atribuindo $x=\left(V_{1}, \ldots, V_{n b}, \theta_{1}, \ldots, \theta_{n b}\right) \in \mathbb{R}^{2 n b}$ às variáveis, o problema central deste trabalho pode ser modelado como um problema de mínimos quadrados não-linear restrito,

$$
\begin{array}{ll}
\min & \frac{1}{2}\|F(x)\|^{2} \\
\text { s.a. } & h(x)=0,
\end{array}
$$

em que $F: \mathbb{R}^{n} \rightarrow \mathbb{R}^{p}, h: \mathbb{R}^{n} \rightarrow \mathbb{R}^{m}$ e $p+m=n$. Se o sistema elétrico estiver operando dentro dos limites de demanda, o problema se resume a encontrar $x^{*}$ tal que

$$
\left[\begin{array}{c}
F\left(x^{*}\right) \\
h\left(x^{*}\right)
\end{array}\right]=0
$$


$\mathrm{Na}$ sequência, apresentamos o procedimento usado para resolver o problema (2.1), o qual baseia-se nos métodos de Lagrangeano Aumentado [4, 5, 9] e LevenbergMarquardt para sistemas não-lineares [6, 8].

\section{O Método Corretivo para as Equações da Rede Elétrica - Lagrangeano Aumentado}

Nesta seção, apresentamos as principais idéias sobre o método proposto. Grande parte das idéias aqui colocadas estão baseadas em recentes avanços na teoria de Lagrangeano Aumentado, contemplando, entre outras coisas, a idéias do método de Levenberg-Marquardt para as iterações internas.

Vamos definir a função Lagrangeana $\mathcal{L}_{A}: \mathbb{R}^{n} \times \mathbb{R}^{m} \times \mathbb{R}^{++} \rightarrow \mathbb{R}$ para o problema $(2.1)$

$$
\mathcal{L}_{A}(x, \lambda, \rho)=\frac{1}{2}\|F(x)\|^{2}+h(x)^{T} \lambda+\frac{\rho}{2}\|h(x)\|^{2} .
$$

Com base nesta função, vamos apresentar uma versão do algoritmo Lagrangeano Aumentado [4]. Para isso, vamos considerar $\left\{\epsilon_{p}\right\}_{p \in \mathbb{N}}$ e $\left\{v_{p}\right\}_{p \in \mathbb{N}}$ sequências de números reais estritamente positivos convergindo para zero.

\section{Algoritmo 1. Lagrangeano Aumentado - Iteração Externa}

Tome $\rho_{1}>0, x_{0}^{s} \in \mathbb{R}^{n},-\infty<\bar{\lambda}_{\text {min }}<\bar{\lambda}_{\text {max }}<+\infty,\left[\bar{\lambda}_{1}\right]_{i} \in\left[\bar{\lambda}_{\text {min }}, \bar{\lambda}_{\text {max }}\right]$, para $i=1, \ldots, m, 1<\zeta_{1} \leq \zeta_{2} \in(0,1)$ e $\gamma \in(0,1)$.

Passo 0. Faça $l \leftarrow 1$.

Passo 1. Escolha $\tau_{l}=\min \left\{\epsilon_{l}, v_{l}\left\|h\left(x_{l}\right)\right\|\right\}$ e, começando com $x_{l-1}^{s}$, encontre $x_{l}$ tal que

$$
\left\|\nabla_{x} \mathcal{L}_{A}\left(x_{l}, \overline{\lambda_{l}}, \rho_{l}\right)\right\| \leq \tau_{l} .
$$

Se $h\left(x_{l}\right)=0$ e $\nabla_{x} \mathcal{L}_{A}\left(x_{l}, \bar{\lambda}_{l}, \rho_{l}\right)=0$ termine o algoritmo declarando que $x_{l}$ é um ponto estacionário de (2.1) com multiplicador de Lagrange $\bar{\lambda}_{l}$ associado.

Passo 2. Estime o vetor dos multiplicadores de Lagrange por

$$
\lambda_{l+1}=\bar{\lambda}_{l}+\rho_{l} h\left(x_{l}\right),
$$

e escolha

$\left[\bar{\lambda}_{l+1}\right]_{i} \in\left[\bar{\lambda}_{\min }, \bar{\lambda}_{\max }\right]$, para $i=1, \ldots, m$.

Passo 3. Se $\left\|h\left(x_{l}\right)\right\|_{\infty} \leq \gamma\left\|h\left(x_{l-1}\right)\right\|_{\infty}$ então

$$
\rho_{l+1} \leftarrow \rho_{l},
$$

senão, escolha

$$
\rho_{l+1} \in\left[\zeta_{1} \rho_{l}, \zeta_{2} \rho_{l}\right]
$$

Passo 4. Faça $x_{l}^{s}=x_{l}, l \leftarrow l+1$

e volte para o Passo 1. 
Além da vantagem de resolver um problema restrito por uma sequência de problemas irrestritos (ou pelo menos problemas mais simples que o original, ver [1]), o método Lagrangeano Aumentado apresenta ainda boas propriedades de convergência, conforme colocamos na sequência. Considerando $\left\{x_{k}\right\}$ a sequência gerada pelo Algoritmo 1 e $x^{*}$ um de seus pontos de acumulação, os seguintes resultados se verificam $[1,5]$ :

(i) $x^{*}$ é um ponto estacionário de

$$
\min \quad\|h(x)\|^{2} \quad \text { s.a. } \quad x \in \mathbb{R}^{n}
$$

(ii) se $x^{*}$ é viável $\left(h\left(x^{*}\right)=0\right)$ e o Jacobiano $h^{\prime}\left(x^{*}\right) \in \mathbb{R}^{m \times n}$ tem posto completo, então $x^{*}$ satisfaz as condições Karush-Kuhn-Tucker do problema (2.1), ou seja, é um ponto estacionário do problema original.

Em cada iteração $l$ do método de Lagrangeano Aumentado, que chamaremos de iteração externa, precisamos encontrar $x_{l}$ que satisfaça (3.3). A fase para obter esta solução aproximada $x_{l}$ chamaremos de iteração interna.

\subsection{A iteração interna}

No Passo 1 do Algoritmo 1 precisamos encontrar $x_{l}$ que satisfaça a condição (3.3). Uma alternativa para obter $x_{l}$ que satisfaça esta condição consiste em minimizar a função Lagrangeana (3.2) em $\mathbb{R}^{n}$.

Neste trabalho, iremos tirar proveito das características peculiares do problema (2.1), obtendo um algoritmo orientado para a fase interna. Para isso, observe que

$$
\begin{gathered}
\mathcal{L}_{A}(x, \lambda, \rho)=\frac{1}{2}\|F(x)\|^{2}+h(x)^{T} \lambda+\frac{\rho}{2}\|h(x)\|^{2} \\
=\frac{1}{2}\|F(x)\|^{2}+\frac{1}{2}\left\|\sqrt{\rho} h(x)+\frac{\lambda}{\sqrt{\rho}}\right\|^{2}-\frac{1}{2 \rho}\|\lambda\|^{2} \\
=\frac{1}{2}\left\|\left[\begin{array}{c}
F(x) \\
\sqrt{\rho} h(x)
\end{array}\right]+\left[\begin{array}{c}
0 \\
\frac{\lambda}{\sqrt{\rho}}
\end{array}\right]\right\|^{2}-\frac{1}{2 \rho}\|\lambda\|^{2} .
\end{gathered}
$$

Então, uma vez fixado $\lambda_{l} \in \mathbb{R}^{m}$ e $\rho_{l} \in \mathbb{R}^{++}$, temos que

$$
\min _{x \in \mathbb{R}^{n}} \quad \mathcal{L}_{A}\left(x, \lambda_{l}, \rho_{l}\right)
$$

é equivalente a

$$
\min _{x \in \mathbb{R}^{n}} \frac{1}{2}\left\|\left[\begin{array}{c}
F(x) \\
\sqrt{\rho_{l}} h(x)
\end{array}\right]+\left[\begin{array}{c}
0 \\
\frac{\lambda_{l}}{\sqrt{\rho_{l}}}
\end{array}\right]\right\|^{2},
$$

que é um problema de mínimos quadrados não-linear.

Para resolver o problema (3.4) vamos observar o sistema não-linear associado e abordá-lo pelo método de Levenberg-Marquardt [6, 8, 13], embora outros algoritmos para este problema possam também serem empregados. Com estas considerações 
não serão necessárias informações de segunda ordem do problema na iteração interna.

Vamos descrever o procedimento de um passo de uma iteração interna: Considere o sistema de equações não-lineares $G: \mathbb{R}^{n} \rightarrow \mathbb{R}^{p+m}$,

$$
G(x)=\left[\begin{array}{c}
F(x) \\
\sqrt{\rho_{l}} h(x)
\end{array}\right]+\left[\begin{array}{c}
0 \\
\frac{\lambda_{l}}{\sqrt{\rho_{l}}}
\end{array}\right],
$$

e denote por $G^{\prime}(x) \in \mathbb{R}^{(p+m) \times n}$ o Jacobiano de $G$ avaliado no ponto $x \in \mathbb{R}^{n}$. Como em cada iteração externa precisamos calcular (3.3), é conveniente expressar esse gradiente em termos da aplicação $G$ :

$$
\nabla_{x} \mathcal{L}_{A}\left(x, \lambda_{l}, \rho_{l}\right)=G^{\prime}(x)^{T} G(x) .
$$

Para descrever o procedimento considere $y_{k}$ um iterado da iteração interna. Uma iteração do método Levenberg-Marquardt encontra uma direção $d_{k}^{L M} \in \mathbb{R}^{n}$ minimizando o modelo quadrático de Gauss-Newton para $G$ acrescido de um termo de regularização, ou seja,

$$
d_{k}^{L M}=\arg \min _{d \in \mathbb{R}^{n}}\left\|G^{\prime}\left(y_{k}\right) d+G\left(y_{k}\right)\right\|^{2}+\mu_{k}\|d\|^{2},
$$

em que $\mu_{k}>0$, denominado parâmetro Levenberg-Marquardt, é um escalar convenientemente escolhido. Por conseguinte, o método prossegue fazendo uma busca linear na direção $d_{k}^{L M}$ a partir de $y_{k}$.

Impondo as condições necessárias de primeira ordem para o problema (3.6), temos que $d_{k}^{L M}$ é solução do sistema linear

$$
\left(G^{\prime}\left(y_{k}\right)^{T} G^{\prime}\left(y_{k}\right)+\mu_{k} I\right) d=-G^{\prime}\left(y_{k}\right)^{T} G\left(y_{k}\right) .
$$

Note que, para resolver o sistema acima, precisamos da matriz $G^{\prime}\left(y_{k}\right)^{T} G^{\prime}\left(y_{k}\right) \in$ $\mathbb{R}^{n \times n}$, o que nem sempre é uma prática usual em virtude da dimensão e estrutura de $G^{\prime}\left(y_{k}\right)$. Além disso, o número de condição da matriz $G^{\prime}\left(y_{k}\right)^{T} G^{\prime}\left(y_{k}\right)$ é o quadrado do número de condição da matriz $G^{\prime}\left(y_{k}\right)$ [7].

Uma abordagem eficaz para contornar esta dificuldade consiste em considerar o problema (3.6) como um problema de mínimos quadrados linear (PQML) [7], ou seja,

$$
\min _{d \in \mathbb{R}^{n}}\left\|\left[\begin{array}{c}
G^{\prime}\left(y_{k}\right) \\
\sqrt{\mu_{k}} I
\end{array}\right] d+\left[\begin{array}{c}
G\left(y_{k}\right) \\
0
\end{array}\right]\right\|^{2} .
$$

A partir desta formulação, pode-se usar procedimentos adequados aproveitando a estrutura da matriz

$$
\left[\begin{array}{c}
G^{\prime}\left(y_{k}\right) \\
\sqrt{\mu_{k}} I
\end{array}\right] \in \mathbb{R}^{(p+m+n) \times n} .
$$

Apesar da simplicidade, o método de Levenberg-Marquardt apresenta propriedades de convergência bem favoráveis, garantindo, em cada iteração interna, convergência para um ponto estacionário do problema $(3.4)[8,6,13]$ e, consequentemente, o cumprimento da tolerância (3.3) em algum passo finito da iteração interna. 
A escolha do parâmetro $\mu_{k}$ : O parâmetro $\mu_{k}$ no método Levenberg-Marquardt tem influência essencial na convergência dos iterados. Para o caso em que a sequência da iteração interna $\left\{y_{k}\right\}$ converge para um ponto $y_{*}$ que seja raiz do sistema nãolinear (3.5), uma escolha razoável para uma iteração $k$ é $\mu_{k}=\mu\left\|G\left(y_{k}\right)\right\|$, para algum $\mu>0$. Sob hipóteses usuais, entre elas $G\left(y_{*}\right)=0$, prova-se que com esta escolha a sequência dos iterados $\left\{y_{k}\right\}$ apresenta convergência localmente quadrática [6]. Entretanto, como também estamos admitindo convergência para um ponto $y_{*}$ que não seja uma raíz de $G$, será incorporada a filosofia de Barzilai-Borwein $[3,4,11]$ na escolha do parâmetro $\mu_{k}$. Portanto, fixado $\bar{\mu}_{2}>0$, o parâmetro Levenberg-Marquardt será escolhido como

$$
\mu_{k}=\min \left\{\bar{\mu}_{2}\left\|G\left(y_{k}\right)\right\|, \mu_{\text {aux }}\right\},
$$

em que $\mu_{a u x}$ é obtido baseado nas idéias de Birgin e Martínez [4]. Assim, fixados $0<\sigma_{\min }<\sigma_{\max }$

$$
\mu_{\text {aux }}=\max \left\{\sigma_{\min }, \min \left\{\sigma_{\max }, \sigma_{k}\right\}\right\},
$$

em que $\sigma_{k}$ é escolhido de maneira que a matriz $\left(G^{\prime}\left(y_{k}\right)^{T} G\left(y_{k}\right)+\sigma_{k} I\right)$ esteja o mais próximo possível de satisfazer a equação secante [9]. Consequentemente, conhecendo dois iterados consecutivos de uma dada iteração interna, digamos $y_{k-1}$ e $y_{k}$, e definindo

$$
z_{k}=G^{\prime}\left(y_{k}\right)^{T} G\left(y_{k}\right)-G^{\prime}\left(y_{k-1}\right)^{T} G\left(y_{k-1}\right)
$$

e

$$
s_{k}=y_{k}-y_{k-1},
$$

temos que

$$
\sigma_{k}=\underset{\sigma \in \mathbb{R}}{\arg \min }\left\|\left(G^{\prime}\left(y_{k}\right)^{T} G\left(y_{k}\right)+\sigma I\right) s_{k}-y_{k}\right\|^{2} .
$$

Deste problema obtemos uma expressão fechada para o parâmetro $\sigma_{k}$ :

$$
\begin{aligned}
\sigma_{k} & =\frac{\left(z_{k}-G^{\prime}\left(y_{k}\right)^{T} G^{\prime}\left(y_{k}\right) s_{k}\right)^{T} s_{k}}{s_{k}^{T} s_{k}} \\
& =\frac{z_{k}^{T} s_{k}-\left\|G^{\prime}\left(y_{k}\right) s_{k}\right\|^{2}}{s_{k}^{T} s_{k}} .
\end{aligned}
$$

O algoritmo da iteração interna é descrito com se segue. Para isso, vamos presumir que estamos em uma iteração externa $l$, e, portanto, são conhecidos $x_{l-1}^{s} \in$ $\mathbb{R}^{n}, \tau_{l}, \lambda_{l}$ e $\rho_{l}$.

Algoritmo 2 (Iteração Interna). Tome $\bar{\mu}_{1}, \bar{\mu}_{2}>0,0<\sigma_{\min }<\sigma_{\max }<+\infty$, $\eta_{1}, \eta_{2}, \beta \in(0,1)$. Faça $y_{0} \leftarrow x_{l-1}^{s}$ e $k \leftarrow 0$.

Passo 1. Considere $G$ como em (3.5) e calcule $G\left(y_{k}\right)$ e $G^{\prime}\left(y_{k}\right)$. Se $\left\|G^{\prime}\left(y_{k}\right) G\left(y_{k}\right)\right\| \leq$ $\tau_{l}$ então pare a iteração interna e faça $x_{l}^{s} \leftarrow y_{k}$ (satisfazendo (3.3)).

Passo 2. Se $k=0$ faça $\sigma_{k}=\bar{\mu}_{1}\left\|G\left(y_{k}\right)\right\|$. Senão, obtenha $z_{k}$ e $s_{k}$ como em (3.8) e (3.9), respectivamente, e calcule

$$
\sigma_{k}=\frac{z_{k}^{T} s_{k}-\left\|G^{\prime}\left(y_{k}\right) s_{k}\right\|^{2}}{s_{k}^{T} s_{k}}
$$


Passo 3. Escolha de $\mu_{k}$ : Considere $\mu_{a u x}=\min \left\{\max \left\{\sigma_{\min }, \sigma_{k}\right\}, \sigma_{\max }\right\}$ e faça

$$
\mu_{k}=\min \left\{\bar{\mu}_{2}\left\|G\left(y_{k}\right)\right\|, \mu_{\text {aux }}\right\} .
$$

Passo 4. Encontre $d_{k}^{L M}$ solução do PQML:

$$
\underset{d \in \mathbb{R}^{n}}{\arg \min }\left\|G^{\prime}\left(y_{k}\right) d+G\left(y_{k}\right)\right\|^{2}+\mu_{k}\|d\|^{2},
$$

Passo 5. Se $\left\|G\left(y_{k}+d_{k}^{L M}\right)\right\| \leq \eta_{1}\left\|G\left(y_{k}\right)\right\|$ faça $t_{k}=1$. Senão, calcule $t_{k}=\arg \max \left\{\beta^{i} \mid i=1,2, \ldots\right\}$ tal que

$$
\left\|G\left(y_{k}+t_{k} d_{k}^{L M}\right)\right\|^{2} \leq\left\|G\left(y_{k}\right)\right\|^{2}+2 t_{k} \eta_{2}\left(d_{k}^{L M}\right)^{T} G^{\prime}\left(y_{k}\right)^{T} G\left(y_{k}\right) .
$$

Passo 6. Faça $y_{k+1}=y_{k}+t_{k} d_{k}^{L M}, k \leftarrow k+1$ e volte para o Passo 1.

Vale a pena ressaltar que o Passo 5 do algoritmo acima é uma busca linear na direção $d_{k}^{L M}$. Com este procedimento, garantimos convergência para pontos estacionários do problema (3.4) [6, 8], e, consequentemente, asseguramos que a condição (3.3) do Passo 1 da iteração externa (Algoritmo 1) será satisfeita após um numero finito de iterações do Algoritmo 2.

\section{Resultados Numéricos}

O objetivo desta seção é apresentar os testes computacionais realizados com método o proposto. Os testes referem-se a problemas padrões IEEE [12] em sistemas de potências, conforme listados na Tabela 2. O sistema SSB 340 corresponde ao sistema simplificado Sul-Sudeste brasileiro [2]. A implementação foi desempenhada no ambiente Matlab 7.0 [10].

Os parâmetros do algoritmo, tanto para a iteração externa como para a interna, são apresentados a seguir. Sempre que possível, procuramos usar valores sugeridos nos artigos especializados $[4,6,8]$.

Parâmetros para iteração externa: $\left\{\epsilon_{p}\right\}=\{v\}_{p}=\left\{10^{-1} / 2^{p}\right\}_{p \in \mathbb{N}}, \rho_{1}=100, \bar{\lambda}_{\text {min }}=$ $-10^{10}, \bar{\lambda}_{\text {max }}=10^{10} x_{0}^{S}=(1, \ldots, 1)^{T}, \lambda_{1}=(0, \ldots, 0)^{T}, \zeta_{1}=\zeta_{2}=10$ e $\gamma=0.25$. Tolerância para admitir a convergência: $\left\|\nabla \mathcal{L}\left(x_{*}, \lambda_{*}\right)\right\|_{\infty} \leq 10^{-3}$.

A atualização de $\bar{\lambda}_{l+1}$ na iteração externa (Algoritmo 1) foi feita resolvendo-se o problema de mínimos quadrados linear,

$$
h^{\prime}\left(x_{l}\right)^{T} \bar{\lambda}_{l+1}=-F^{\prime}\left(x_{l}\right)^{T} F\left(x_{l}\right) .
$$

Parâmetros para iteração interna: $\mu_{1}=10^{-5} \mu_{2}=10, \sigma_{\min }=5 \times 10^{-4}, \sigma_{\max }=$

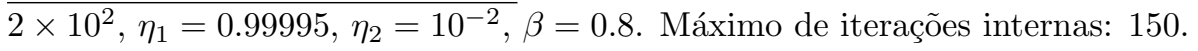


Tabela 1: Dimensão dos problemas testados.

\begin{tabular}{lccc}
\hline \hline & \multicolumn{3}{c}{ Número de Variáveis } \\
Problema & $n$ & $p$ & $m$ \\
\cline { 2 - 4 } IEEE6 & 9 & 7 & 2 \\
IEEE14 & 22 & 20 & 2 \\
IEEE30 & 53 & 41 & 12 \\
IEEE57 & 106 & 74 & 32 \\
IEEE118 & 201 & 181 & 20 \\
SSB340 & 626 & 420 & 206 \\
\hline \hline
\end{tabular}

Para efeito de validação do algoritmo, comparamos o método proposto neste trabalho com o método de Newton com busca linear [9] aplicado diretamente à função Lagrangeana do problema (2.1),

$$
\mathcal{L}(x, \lambda)=\frac{1}{2}\|F(x)\|^{2}+\lambda^{T} h(x) .
$$

Neste caso, a direção de Newton $d_{k}^{N} \in \mathbb{R}^{n+m}$ é obtida resolvendo-se o sistema linear,

$$
W_{k} d=-\nabla \mathcal{L}\left(x_{k}, \lambda_{k}\right)
$$

em que

$$
W_{k}=\nabla^{2} \mathcal{L}\left(x_{k}, \lambda_{k}\right) \in \mathbb{R}^{(n+m) \times(n+m)} .
$$

Desta forma, a partir de iterados $x_{k}$ e $\lambda_{k}$, o método de Newton procede fazendo uma busca linear na direção $d_{k}^{N}$, com parâmetros iguais aos do Passo 5 no Algoritmo 2. Admitimos um número máximo de 150 iterações de Newton.

Os resultados estão apresentados na Tabela 2. A nomenclatura NIT representa o número total de iterações. Assim, $a\left(i_{1}, i_{2}\right)$ significa um total de $a$ iterações, ou seja, $a=i_{1}+i_{2}$, em que $i_{1}$ é o número de iterações na primeira iteração interna e $i_{2}$ o da segunda iteração interna. Para representar o número de avaliações da função de mérito [9] na busca linear usamos NAV.

Podemos observar nos resultados que o método proposto sempre converge para a solução obtida por Newton. Esta é uma característica relevante, pois métodos que usam apenas informação de primeira ordem nem sempre são robustos do ponto de vista de convergência. Nos Problemas IEEE57 e SSB340 a direção de Newton e a direção do gradiente tendem a ortogonalidade em virtude da matriz Hessiana do Lagrangeano, $W_{k}$, tornar-se excessivamente mal-condicionada.

Embora não seja o objetivo do trabalho comparar desempenhos, observamos que, apesar do número razoável de avaliação da função, o método proposto mostrouse competitivo em sistemas de pequena e média dimensão, sendo promitente a realização de experimentos em problemas reais, onde as dimensões são maiores. 
Tabela 2: Desempenho do método.

\begin{tabular}{|l|lccc|}
\hline \hline & \multicolumn{4}{|c|}{ Método Proposto } \\
Problema & NIT $^{\dagger}$ & NAV & $\left\|F\left(x_{*}\right)\right\|$ & $\left\|\nabla \mathcal{L}\left(x_{*}, \lambda_{*}\right)\right\|$ \\
\hline IEEE6 & $11(9,2)$ & 38 & $1.8 \times 10^{-1}$ & $5.7 \times 10^{-5}$ \\
IEEE14 & $6(6)$ & 11 & $9.4 \times 10^{-2}$ & $8.2 \times 10^{-4}$ \\
IEEE30 & $8(8)$ & 20 & $3.5 \times 10^{-2}$ & $6.2 \times 10^{-4}$ \\
IEEE57 & $11(9,2)$ & 19 & $1.9 \times 10^{-2}$ & $6.5 \times 10^{-4}$ \\
IEEE118 & $24(24)$ & 133 & $4.5 \times 10^{-1}$ & $5.9 \times 10^{-4}$ \\
SSB340 & $77(70,7)$ & 328 & 1.6 & $3.2 \times 10^{-4}$ \\
\hline \hline
\end{tabular}

$\dagger a(b, c)$ representa $b$ iterações na primeira iteração interna, $c$ iterações na segunda iteração interna e assim sucessivamente, correspondendo ao total de $c(=a+b)$ iterações.

\begin{tabular}{|l|lccc|}
\hline \hline & \multicolumn{4}{|c|}{ Método de Newton } \\
Problema & NIT & NAV & $\left\|F\left(x_{*}\right)\right\|$ & $\left\|\nabla \mathcal{L}\left(x_{*}, \lambda_{*}\right)\right\|$ \\
\hline IEEE6 & 7 & 7 & $1.8 \times 10^{-1}$ & $5.3 \times 10^{-6}$ \\
IEEE14 & 10 & 10 & $9.4 \times 10^{-2}$ & $2.6 \times 10^{-4}$ \\
IEEE30 & 8 & 8 & $2.9 \times 10^{-2}$ & $6,5 \times 10^{-5}$ \\
IEEE57 & Max & - & $2.6 \times 10^{-1}$ & $3.5 \times 10^{-1}$ \\
IEEE118 & 12 & 16 & $4.5 \times 10^{-1}$ & $2.2 \times 10^{-4}$ \\
SSB340 & Max & - & 33.4 & 93.9 \\
\hline \hline
\end{tabular}

\section{Conclusões}

A metodologia proposta neste trabalho apresentou resultados robustos em termos de convergência à solução dos modelos testados. É importante enfatizar que, embora em alguns problemas realize mais iterações que o método de Newton, sua formulação não exige o cálculo da matriz Hessiana e os sistemas lineares envolvidos são de dimensão menores e com estrutura favorável. Além disso, começando com $\bar{\lambda}_{1}=$ $(0, \ldots, 0)^{T} \in \mathbb{R}^{m}$ no Algoritmo 1 e assumindo que o ponto de acumulação $x^{*}$ é uma raíz de $F$, o método se resume ao Levenberg-Marquardt usual, apresentando neste caso convergência localmente quadrática.

Testes numéricos adicionais e otimização da implementação direcionando para aplicações em sistemas de grande porte, podem, eventualmente, mostrar a efetividade do método proposto em relação às técnicas usuais em sistemas de potências.

Em trabalhos futuros, pretendemos incorporar informações de segunda ordem baseadas em atualizações secantes nos modelos envolvidos. Neste caso, a formulação do problema de mínimos quadrados não-linear (2.1) representaria com mais fidelidade o problema central. 


\section{Referências}

[1] R. Andreani, E.G. Birgin, J.M. Martínez, M.L. Schuverdt. Augmented Lagrangian methods with general lower-level constraints, SIAM Journal on Optimization, to appear.

[2] L.V. Barbosa, "Análise e Desenvolvimento de Metodologias Corretivas para a Restauração da Solução das Equações da Rede Elétrica". Tese de Doutorado. Dept Eng. Elétrica, Universidade Ferderal de Sta. Catarina, 2001.

[3] J. Barzilai, J.M. Borwein. Two point step size gradient methods, IMA Journal of Numerical Analysis, 8 (1988), 141-148.

[4] E.G. Birgin, J.M. Martínez, Structured minimal-memory inexact quasi-Newton method and secant preconditioners for augmented Lagrangian optimization, Computational Optimization and Applications, 39, No. 1 (2008), 1-16.

[5] A.R. Conn, N.I.M. Gould, Ph.L. Toint, A globally convergent Augmented Lagrangian algorithm for optimization with general constraints and simple bounds, SIAM Journal on Numerical Analysis, 28 (1991), 545-572.

[6] J. Fan, Y. Yuan. On the quadratic convergence of the Levenberg-Marquardt method without nonsingularity assumption, Computing, 74 (2005), 23-39.

[7] G.A. Golub, C.F. Van Loan, "Matrix Computations", 3rd. Edition, The John Hopkins University Press, London, 1996.

[8] C. Kanzow, N. Yamashita, M. Fukushima, Levenberg-Marquardt methods with strong local convergence properties for solving nonlinear equations with convex constraints, Journal of Computational and Applied Mathematics, 174, No. 2, (2004), 375-397.

[9] J. Nocedal, S.J. Wright, "Numerical Optimization", Springer Series in Operations Research, Springer Verlag, New York, 1999.

[10] E. Pärt-Enander, A. Sjöberg, "The Matlab 5 Handbook", Addison Wesley, Harlow, UK, 1999.

[11] M. Raydan, The Barzilai and Borwein gradient method for large scale unconstrained minimization problem, SIAM Journal on Optimization, 7 (1997), 26-33.

[12] B. Wollenberg, A. Wood, "Power Generation, Operation and Control", Wiley, 2nd ed., New York, 1996.

[13] N. Yamashita, M. Fukushima, On the rate of convergence of the LevenbergMarquardt method, Computing, 15, (2001), 239-249. 